\title{
Age-Related Trajectories of Functional Coupling between the VTA and Nucleus Accumbens Depend on Motivational State
}

\author{
마shnu P. Murty, ${ }^{1}$ Hemali Shah, ${ }^{3}$ David Montez, ${ }^{2}$ Will Foran, ${ }^{2}{ }^{-}$Finnegan Calabro, ${ }^{2,4}$ and ${ }^{\circledR B}$ Beatriz Luna ${ }^{2,5,6}$ \\ ${ }^{1}$ Department of Psychology, Temple University, Philadelphia, Pennsylvania 19122, Departments of ${ }^{2}$ Psychiatry, ${ }^{3}$ Neuroscience, ${ }^{4}$ Biomedical Engineering, \\ ${ }^{5}$ Psychology, University of Pittsburgh, Pittsburgh, Pennsylvania 15213, and ${ }^{6}$ Center for the Neural Basis of Cognition, Pittsburgh, Pennsylvania 15213
}

Over-engagement of the mesolimbic dopamine system is thought to enhance motivation in adolescents. Whereas human neuroimaging has characterized event-evoked responses of the mesolimbic system in adolescents, research has yet to characterize state-dependent engagement (i.e., seconds to minutes) of this system in goal-relevant contexts. In the current longitudinal study, we characterized age-related changes in state-dependent coupling in male and female human participants ranging in age from adolescence to adulthood. Analyses focused on two key regions of the mesolimbic dopamine system, the ventral tegmental area (VTA) and nucleus accumbens (NAcc). Although there were no differences in VTA-NAcc functional coupling in a resting-state context, VTA-NAcc functional coupling was enhanced in preadolescence/early adolescence and decreased into adulthood in a motivational context, in which individuals had to translate goal-relevant cues into instrumental actions. Furthermore, we found that task-related activation in orbitofrontal cortex, middle temporal gyrus, and visual association cortex partially mediated age-related changes in state-dependent VTA-NAcc functional coupling. These results extend prior models of neurodevelopment by showing a relationship between cortical event-evoked activation and statedependent increases in subcortical engagement of mesolimbic systems.

Key words: adolescence; fMRI; longitudinal; motivation; nucleus accumbens; VTA

Significance Statement

Adolescence is characterized by increased motivated behavior, which is thought to result from an over-engagement of mesolimbic dopamine systems. Rodent models show increases in state-dependent engagement of mesolimbic systems in adolescence. However, human neuroimaging research has mainly focused on event-evoked responses (i.e., reward cues). We show that in motivational contexts, there is increased state-dependent coupling across mesolimbic systems in preadolescence/early adolescence that decreases into adulthood and is further predicted by event-evoked cortical responses. Critically, these developmental trajectories were specific to motivationally relevant contexts and were not apparent during resting state. These findings extend emerging models of human development and suggest that state-dependent increases in dopamine signaling may underlie heightened motivation.

\section{Introduction}

Adolescence is characterized by increased motivation, which is thought to promote risk-taking behaviors that can undermine survival (Luna et al., 2015; Shulman et al., 2016). Increased motivation and initiation of goal-oriented behaviors in adolescence may result from an over-engagement of mesolimbic dopamine systems (Luna et al., 2015; Shulman et al., 2016), including projections from the ventral tegmental area (VTA) to the nucleus accumbens (NAcc). In rodents, the mesolimbic dopamine sys-

\footnotetext{
Received Dec. 12, 2017; revised June 21, 2018; accepted July 9, 2018.

Author contributions: V.P.M., D.M., F.C., and B.L. designed research; V.P.M., H.S., and B.L. performed research;

V.P.M., H.S., D.M., W.F., and F.C. analyzed data; V.P.M. and B.L. wrote the paper.

The authors declare no competing financial interests.

Correspondence should be addressed to Vishnu P. Murty, Department of Psychology, Temple University, Philadelphia, Pennsylvania 19122. E-mail: vishnu.murty@temple.edu.

DOI:10.1523/JNEUROSCI.3508-17.2018

Copyright $\odot 2018$ the authors $\quad 0270-6474 / 18 / 387420-08 \$ 15.00 / 0$
}

tems fire more strongly in response to rewards in adolescents versus adults (Wahlstrom et al., 2010a,b), which has also been shown in humans (Padmanabhan and Luna, 2014; Silverman et al., 2015). In rodents, adolescents also show increased sustained engagement of mesolimbic systems (i.e., tonic dopamine firing). In humans, less is known about the neurodevelopment of statedependent interactions between the VTA and NAcc. The current study characterizes longitudinal neurodevelopment trajectories of VTA-NAcc interactions across motivation (operationalized as a context in which individuals translated goal-relevant cues into instrumental actions) and resting-state contexts.

A growing body of human neuroimaging has detailed changes in mesolimbic systems unique to adolescence (Padmanabhan and Luna, 2014; Murty et al., 2016; Telzer, 2016), including increased engagement of regions within the mesolimbic system during motivated behaviors that peaks during adolescence (Silverman et al., 2015). Research has also shown that NAcc responsivity 
relates to a variety of goal-oriented behaviors in adolescence (Chein et al., 2011; van Duijvenvoorde et al., 2014; Hawes et al., 2017). Although this research has characterized human NAcc responses in adolescents, open questions remain regarding the role of the VTA in adolescent neurodevelopment.

Animal models of mesolimbic systems have characterized the mechanistic underpinning of increased motivation, showing that increased tonic dopamine signaling can increase sensitivity to salient stimuli, such as rewards (Grace et al., 2007; Grace, 2016). Specifically, when animals are in motivationally relevant contexts, there is greater prolonged, baseline firing of the VTA. In turn, increased baseline firing across VTA and NAcc results in greater behavioral activation and motivated behavior (Shohamy and Adcock, 2010; Norbury and Husain, 2015). In regards to development, animal models posit that prolonged, context-dependent increases in dopamine availability and signaling in adolescence increase motivation and the initiation of goal-oriented behaviors (Wahlstrom et al., 2010a,b). Many facets of these animal models of development, particularly state-dependent increases in mesolimbic systems, have yet to be tested in humans.

Methodological advancements in human neuroimaging allow for the characterization of state-dependent functional coupling. "Background connectivity" analyses have been developed to investigate context-dependent changes in network connectivity over prolonged time scales (Al-Aidroos et al., 2012; NormanHaignere et al., 2012; Duncan et al., 2014). This approach removes variance associated with event-evoked activity from neuroimaging data, thus allowing for the characterization of state-dependent shifts in connectivity extending across entire task contexts. Accordingly, this approach measures changes in network connectivity persisting over an entire behavioral context (i.e., seconds to minutes) rather than in response to discrete cues (i.e., milliseconds to seconds).

A secondary goal of the study was to characterize how cortical detection of task-related cues influences changes in background connectivity throughout development. Prior work has suggested that cortical inputs in response to task-related cues (i.e., phasiclike responses) regulates VTA engagement (Ballard et al., 2011; Murty et al., 2017). Furthermore, recent rodent research shows that phasic increases in dopamine signaling can increase statedependent engagement of the mesolimbic system for prolonged periods of time (Lohani et al., 2018). These findings suggest that age-related differences in the cortical detection of task-relevant cues may be associated with downstream engagement of statedependent functional coupling.

The current study characterizes neurodevelopmental trajectories of state-dependent changes in VTA-NAcc functional coupling, a putative indirect marker of functional connectivity within the mesolimbic dopamine system. Using a fast, longitudinal design, we acquired functional magnetic resonance imaging (fMRI) data during resting state and a motivation context (a task with rewards, loss, and neutral trials) on participants ranging from adolescence to early adulthood. Critically, we operationalize our motivational contexts as a general state in which participants are initiated goal-oriented behaviors, rather than a context specific to reward incentives. We implemented background connectivity techniques to characterize changes in VTA-NAcc connectivity that reflect state-dependent, rather than event-evoked, engagement. We also examined how event-evoked reactivity in response to motivationally relevant cues related to age-related changes in VTA-NAcc state-dependent coupling. We hypothesized that VTA-NAcc coupling in the motivated context would be greatest during adolescence and decrease into adulthood. Fur- thermore, we hypothesized that these developmental trajectories would be specific to motivation contexts and would be associated with cortical event-evoked responses.

\section{Materials and Methods}

\section{Participants}

Data from 180 participants were analyzed (84 females; age range, 10.130.3 years; median age, 18.1 years). In this longitudinal design, participants completed one to three visits with 106 participants completing one visit, 29 participants completing two visits, and 45 participants completing three visits (total visits, 299). The median interval between visits was 1.6 years (range, $1.0-4.4$ years). Each visit included behavioral assessments in addition to an MRI scan. Participants were recruited from the local population and were screened for psychiatric and neurological problems, medication history, first-degree relatives with major psychiatric illness, and MRI contraindications (e.g., claustrophobia, metal in the body, pregnancy). Participants and their parents (for subjects under the age of 18) gave informed consent, which was approved by the University of Pittsburgh Institutional Review Board. Previous publications have reported on separate research inquiries on subsets of this dataset (Marek et al., 2015; Paulsen et al., 2015; Hawes et al., 2017; Jalbrzikowski et al., 2017).

Resting-state and motivation (rewarded antisaccade) tasks

To characterize context-dependent developmental trajectories, we analyzed fMRI data collected during a resting-state and a motivation task.

During the resting-state scan, participants were instructed to keep their eyes open and fixate on a cross that was displayed on the center of the screen for $5 \mathrm{~min}$.

During the motivation task, participants completed a rewarded antisaccade paradigm as described previously (Paulsen et al., 2015). On each trial, participants first viewed a cue for $1.5 \mathrm{~s}$ indicating whether they could gain points (green bars indicating Reward), lose points (red bars indicating Loss), or neither gain or lose points (gray bars indicating Neutral) for successful performance during a subsequent antisaccade. Participants then viewed a central fixation cross for $1.5 \mathrm{~s}$ during a preparatory period, which was replaced by a small yellow dot placed in one of three unpredictable peripheral locations in the horizontal meridian. Participants were required to saccade to the mirror location of the dot after its presentation. Immediately after the saccade, participants heard a cash register sound for successful performance or a buzzer sound for incorrect responses. This was followed by an intertrial interval varying from 1.5 to $19.5 \mathrm{~s}$ after an exponential distribution. Saccade metrics were obtained using a long-range optics eye-tracking system to determine correct responses. Over the course of four runs of the motivation task, participants completed 56 reward, neutral, and loss trials. In addition to these trials, participants viewed an additional 72 partial trials (i.e., catch trials) in which cues were presented in isolation or in addition to the preparatory period, without presentation of the yellow antisaccade dot. These catch trials allowed us to better model each period of the trial (cue, preparation, antisaccade). More details on the task, incentive structure, and eyetracking software/analysis are described in detail previously (Paulsen et al., 2015).

\section{$M R$ data acquisition and preprocessing}

MRI data were collected using a 3.0T Siemens Trio scanner. fMRI data were collected during the motivation task (rewarded AS) and resting state. For both acquisitions, a single-shot echoplanar imaging sequence sensitive to BOLD contrast (T2*) was collected (TR, $1.5 \mathrm{~s}$; TE, $25 \mathrm{~ms}$; flip angle, $70^{\circ}$; in-plane resolution, $3.125 \mathrm{~mm}$; slice thickness, $4 \mathrm{~mm}$; Anterior Commisure/Posterior Commisure aligned rest, 200 acquisitions; reward, $302 * 4$ runs). In addition, we collected a high-resolution anatomical image (MPRAGE; 192 slices; slice thickness, $1 \mathrm{~mm}$ ) for use in spatial normalization.

Resting-state fMRI data were preprocessed using a pipeline designed to minimize the effects of head motion (Hallquist et al., 2013). This included simultaneous 4D slice-timing and head motion correction, skull stripping, intensity thresholding, wavelet despiking (Patel et al., 2014), coregistration to the MPRAGE, nonlinear warping to MNI space, 
spatial smoothing with a $5 \mathrm{~mm}$ Gaussian kernel, intensity normalization, and nuisance regression based on head motion $\left(6^{\circ}\right.$ of translation/rotation and their first derivative) and non-gray matter signal (white matter and CSF and their first derivative). Bandpass filtering between 0.01875 and $0.08 \mathrm{~Hz}$ was done simultaneously with nuisance regression. Preprocessing of the fMRI data from the motivation task was implemented to parallel the resting-state data with a few modifications (detailed below). fMRI data underwent slice-timing correction, head motion correction, skull stripping, intensity thresholding, wavelet despiking, coregistration/ normalization, spatial smoothing, intensity normalization, nuisance regression, and high-pass filtering as described above. In both datasets, wavelet despiking was used as an alternative to "censoring" or "scrubbing" individual time points associated with head motion. This approach accounts for both high- and low-frequency artifacts that can either be linearly or nonlinearly associated with head motion (www.brainwavelet.org).

To investigate context-related connectivity, as opposed to task-evoked connectivity, we implemented a background connectivity approach (AlAidroos et al., 2012; Duncan et al., 2014). First, we regressed out taskrelated components of the time series using a general linear model (GLM). For each session, we concatenated the time series across the four runs. We concatenated across runs to allow for a better estimation of task-evoked response, which should provide a better estimation of background connectivity. Task-related events were modeled using $3 \mathrm{dDecon}-$ volve in AFNI. Events were modeled using a TENT function to estimate the hemodynamic response for $22.5 \mathrm{~s}$ after each event in $1.5 \mathrm{~s}$ increments. The TENT function, as opposed to using a canonical hemodynamic response function (HRF), does not make assumptions about the shape of HRF responses and thus should more adequately remove task-evoked signals from the time series. We modeled the following task conditions/ events: Neutral Cue, Punishment Cue, Reward Cue, Neutral Preparation, Punishment Preparation, Reward Preparation, Neutral Antisaccade, Punishment Antisaccade, Reward Antisaccade. We also separately modeled incorrect trials across neutral, punishment, and reward conditions as well as trials in which we did not obtain good eye tracking (excluding catch trials). The residuals from this model (i.e., the error term from the GLM) were then bandpass filtered to match the temporal characteristics of the resting-state data.

\section{Experimental design and statistical analysis}

Functional connectivity analysis. To explore functional connectivity in motivation and resting contexts, we calculated pairwise correlations across regions of interest (ROIs) for the time series extracted from resting-state and motivation tasks. ROIs included the VTA, the NAcc, and the caudate. ROIs were defined from probabilistic atlases set to a threshold of 75\% overlap from a probabilistic atlas of the VTA (Murty et al., 2014) and the Harvard-Oxford subcortical probabilistic atlas for the NAcc and caudate (Frazier et al., 2005). Individual $r$ values were then $z$-transformed across visits within each task context. To assess differences in connectivity as a function of age and task context, we implemented a linear mixed-effects model as implemented in MATLAB R2016a with the $z$-transformed correlation value as the dependent variable and condition (rest, motivation), age ${ }^{-1}$, head motion ${ }^{2}$, and sex as fixed-effects factors. To account for the longitudinal nature of the data, we included participant ID, which represents the individual, as a random-effects factor. To investigate the directionality of any age * context interactions, we ran post hoc linear mixed-effects models separately for the rest and incentive data, while still modeling age ${ }^{-1}$, head motion ${ }^{2}$, and sex as fixed-effects factors and participant ID as a random-effects factor. Data were considered significant at $p<0.05$.

We opted to characterize developmental changes with an age ${ }^{-1}$ (i.e., inverse age) rather than a linear age given hypotheses that development is a time period characterized by an initial period of significant growth followed importantly by a period of stabilization into adulthood. Furthermore, previous research from our laboratory has shown that this function provides a better fit than linear age for cognitive development (Ordaz et al., 2013; Simmonds et al., 2017). Further in our sample, statistically age ${ }^{-1}$ resulted in marginally better model fits of the data than linear age (linear: BIC, 1682; inverse: BIC, 1679).
Cue-evoked activation/mediation analysis. To determine how cueevoked activation (period when subjects viewed a colored set of bars indicating potential reward, loss, or neither; see Materials and Methods) related to age-related changes in VTA-NAcc background connectivity during the motivation task, we ran a whole-brain mediation analysis. We hypothesized that cue-evoked activity during the motivation task would mediate age-related trajectories in VTA-NAcc connectivity during the motivation task. We hypothesized that cues initiate motivated behavior and that neural representations of these cues would indicate to increase engagement of mesolimbic systems. To this extent, we wanted to estimate cue-evoked activity collapsed across all three trial types, as all of the cues indicated that individuals were in a motivationally relevant context. We opted to focus our analysis collapsing across the cues types, given that functional coupling between the VTA and NAcc may not be specific to reward sensitivity, but rather to general salience (i.e., any cue that indicates a need for an upcoming behavioral response may engage this circuity). Notably, we did not have any significant results for the analyses detailed below when investigating contrasts of reward $>$ neutral cues, or reward/punishment $>$ neutral cues. Given this, we remodeled our neuroimaging data to optimize extraction of cue-evoked data. For each session, we concatenated the time series across the four runs of the motivation task. We then modeled task-related events using 3dDeconvolve in AFNI. Events were again modeled using a TENT function to estimate the hemodynamic response for $22.5 \mathrm{~s}$ after each event in $1.5 \mathrm{~s}$ increments. We modeled the following task conditions/events collapsed across conditions: Cue (colors representing incentive), Preparation (fixation cross), Correct Antisaccade (saccade to mirror location of target), Incorrect Antisaccade (saccade to target), and Dropped Trials (failure to perform the trial). We then averaged the TENT function for each condition across all individual sessions to generate a voxel-specific HRF. Finally, we computed the dot product of each individual's session-specific HRF to cues against the mean HRF to calculate an estimate of cue-evoked activation.

We wanted to identify neural regions whose cue-evoked activity may mediate the relationship between age and VTA-NAcc connectivity. We ran a group-level analysis running a linear regression against whole-brain cue-evoked activity (masked for gray matter) with age ${ }^{-1}$ and VTA-NAcc connectivity, respectively, across sessions. We thresholded each of these maps at a liberal threshold of $p<0.05$ (cluster extant, 15 voxels) and generated a conjunction mask between these two maps to define ROIs (a more stringent correction was applied to the mediation analysis). This analysis yielded 18 ROIs. To make sure our analysis approach was not overlooking any interesting results that were specific to cues associated with monetary incentives, we re-ran the same analysis using eventevoked activation when comparing reward $>$ neutral and punishment $>$ neutral. However, neither of these analyses yielded significant results.

We then ran a mediation analysis using cue-evoked activation from each of these 18 ROIs as a potential mediator between age ${ }^{-1}$ and VTANAcc connectivity. Mediation analyses were conducted using the Mediation toolbox as implemented in R, explicitly testing whether cue-evoked activity partially or fully mediated the relationship between age ${ }^{-1}$ and VTA-NAcc connectivity. Notably, when estimating cue-evoked activation, differences in background connectivity that extend over the entire time course are captured in the residual term of the GLM, and thus the signals entering our mediation analyses were orthogonal. Significance was tested by computing the significance of the indirect pathway via permutation-based testing and was considered significant at $p<0.0028$ ( $p<0.05$, Bonferroni corrected over 18 ROIs). Notably, participant ID was entered into these models as a random-effects variable, thus accounting for the longitudinal nature of this data analysis.

Brain-behavior correlations. To investigate how VTA-NAcc functional coupling and cue-evoked activation related to motivated behavior, we implemented a linear mixed-effects model as implemented in MATLAB R2016a with motivated performance as the dependent variable, brain activation and age ${ }^{-1}$ as the fixed-effects factor, and participant ID as the random-effects factor. For each participant, motivated behavior was calculated as the difference in accuracy on antisaccade trials preceded by reward versus neutral cue, which has previously been shown to be sensitive to age-related reward effects (Geier et al., 2010). Age ${ }^{-1}$ was 
A

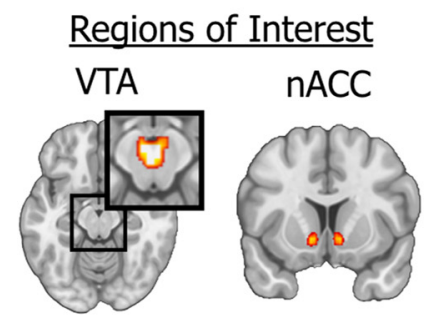

B

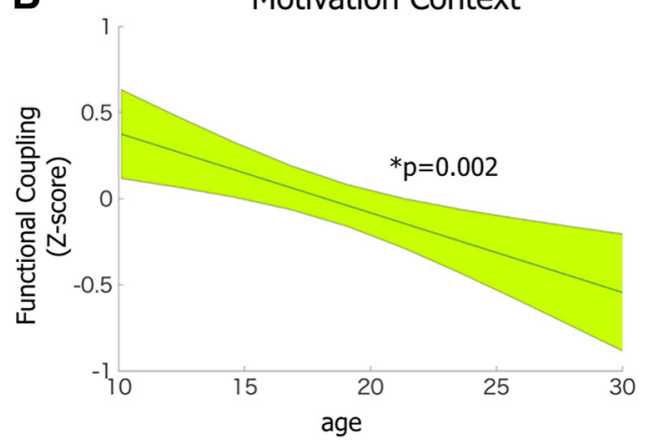

C

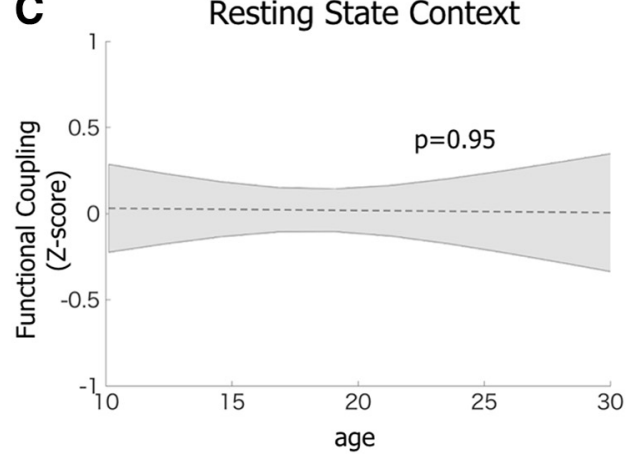

Figure 1. Context-dependent changes in age-related trajectories of VTA-NAcc functional coupling. A, Functional coupling between the VTA and NAcc was assessed in a longitudinal fMRI sample. $B$, In a motivation context, there were age-related decreases in functional coupling between the VTA and NAcc. $C$, There was a nonsignificant relationship between age and functional coupling between the VT and NAcc in the resting-state context. Notably, the plots show the longitudinal trajectory between functional coupling and linear age for visualization purposes; however, the statistics reflect a model in which age was characterized as age ${ }^{-1}$.

Table 1. Age-related effects on VTA-NAcc connectivity and the influence of context

\begin{tabular}{|c|c|c|c|c|c|}
\hline & Estimate & SE & $t$ value & $\mathrm{df}$ & $p$ value \\
\hline \multicolumn{6}{|c|}{ Model 1: Context-dependent effects of age on VTA-NAcc connectivity } \\
\hline Intercept & -1.12 & 0.24 & -4.66 & 591 & $<0.001$ \\
\hline Context & 0.75 & 0.29 & 2.61 & 591 & 0.009 \\
\hline Sex & 0.26 & 0.10 & 2.58 & 591 & 0.01 \\
\hline Motion & 0.07 & 0.04 & 1.74 & 591 & 0.08 \\
\hline Motion $^{2}$ & -0.00002 & 0.002 & -0.01 & 591 & 0.99 \\
\hline $\operatorname{Age}^{-1}$ & 15.12 & 4.02 & 3.76 & 591 & $<0.001$ \\
\hline Context $^{*}$ age $^{-1}$ & -12.64 & 4.78 & -2.64 & 591 & 0.008 \\
\hline \multicolumn{6}{|c|}{ Model 2: Age on VTA-NAcc connectivity during motivation } \\
\hline Intercept & -1.2046 & 0.24237 & -4.9701 & 294 & $<0.001$ \\
\hline Sex & 0.38995 & 0.12922 & 3.0176 & 294 & 0.003 \\
\hline Motio & 0.14087 & 0.075589 & 1.8636 & 294 & 0.06 \\
\hline Motion ${ }^{2}$ & -0.010766 & 0.0074989 & -1.4357 & 294 & 0.15 \\
\hline $\mathrm{Age}^{-1}$ & 14.497 & 4.0805 & 3.5527 & 294 & $<0.001$ \\
\hline \multicolumn{6}{|c|}{ Model 3: Age on VTA-NAcc connectivity during rest } \\
\hline Intercept & -0.28778 & 0.23672 & -1.2157 & 294 & 0.23 \\
\hline Sex & 0.10999 & 0.1146 & 0.95974 & 294 & 0.34 \\
\hline Motion & 0.080531 & 0.056429 & 1.4271 & 294 & 0.15 \\
\hline Motion ${ }^{2}$ & -0.00011999 & 0.0020413 & -0.058779 & 294 & 0.95 \\
\hline $\mathrm{Age}^{-1}$ & 2.2789 & 3.9518 & 0.57666 & 294 & 0.56 \\
\hline
\end{tabular}

Bold font indicates significance at $p<0.05$.

included in these models to account for relationships that were driven purely by collinearity with age rather than variability in neural engagement.

\section{Results}

\section{Motivational context influences VTA-NAcc} developmental trajectories

To test for developmental differences in mesolimbic connectivity in different contexts, we compared functional coupling between the VTA and NAcc (Fig. 1A) as a function of age in resting-state and motivation contexts. Given our interest in context-related differences, we used a background connectivity approach to characterize connectivity during the motivation task. This approach allows us to look at state-dependent differences in connectivity while accounting for any differences in event-evoked activation.

We found a significant interaction between age ${ }^{-1}$ and context (motivation, rest) on VTA-NAcc connectivity $(p=0.008$; Table 1, Model 1). To further characterize this interaction, we separately tested age-related effects on VTA-NAcc connectivity within each context. There was a significant age-related decrease in VTA-NAcc coupling in the motivation context $(p=0.0004$; Fig. 1B; Table 1, Model 2), such that VTA-NAcc coupling was highest in during preadolescence/early adolescence and decreased into adulthood. However, there were no significant agerelated effects in VTA-NAcc coupling during resting state ( $p=$ 0.56; Fig. 1C; Table 1, Model 3).

To test the specificity of our neural findings, we tested differences in connectivity of the VTA with the caudate, a region that is in close spatial proximity to the NAcc but does not receive as many inputs from the VTA (Haber and Knutson, 2010). There were no age ${ }^{\star}$ context interactions $(p=0.63)$ or main effects of age in either context (motivation, $p=0.38$; rest, $p=0.58$ ) in VTA-caudate coupling. These results support that engagement of VTA-NAcc circuits are state dependent and age-related effects are most prominent in motivationally relevant states.

\section{Relationships between age, VTA-NAcc coupling, and cue activation}

We next wanted to draw a link between developmental changes in the ability to interpret motivational cues in the environment and how they relate to VTA-NAcc state-dependent functional coupling. We performed a whole-brain, exploratory analysis to identify neural regions whose cue-evoked activity mediated the relationship between age ${ }^{-1}$ and VTA-NAcc connectivity during the motivation task. First, we identified 18 neural regions that were related to both age and VTA-NAcc functional coupling across sessions (Table 2).

Of these 18 regions, only 3 regions were significant in our mediation analysis $(p<0.05$, Bonferroni corrected). We found that age-related decreases in cue-related activity in the orbitofrontal cortex (OFC; Fig. 2B) and middle temporal gyrus (Fig. $2 C)$ partially mediated age-related differences in VTA-NAcc connectivity. Furthermore, we found that age-related increases in cue-related activity in the visual association cortex (Fig. 2A) partially mediated age-related differences in VTA-NAcc connectivity. Notably, there were small but significant relationships in cue-evoked activation of the middle temporal gyrus with visual association cortex and OFC across participants (Table 3), suggesting that similar mechanisms across regions may be contributing to our mediation effects.

\section{Brain-behavior correlations}

There was not a significant relationship between VTA-NAcc coupling in motivated contexts and motivated behavioral performance (Reward $>$ Neutral; $p=0.98$ ). However, there was a small, but significant, relationship between cue-evoked activation in visual association cortex and motivated behavioral perfor- 
Table 2. Regions showing linear relationships of cue-related activity with age as well as VTA-NAcc connectivity

\begin{tabular}{llllrrr}
\hline & Number of & Relationship & $\begin{array}{l}\text { Relationship } \\
\text { with VTA- }\end{array}$ & & & \\
Region & voxels & with age & NAcc & $x$ & $y$ & $z$ \\
\hline L. fusiform gyrus & 113 & + & - & -38 & -76 & -16 \\
L. middle occipital gyrus & 69 & + & - & -38 & -92 & 4 \\
R. middle occipital gyrus & 53 & + & - & 38 & -86 & 14 \\
L. inferior parietal cortex & 53 & + & - & -28 & -70 & 44 \\
R. fusiform gyrus & 47 & + & - & 32 & -76 & -16 \\
R. insula & 37 & + & - & 34 & 26 & 4 \\
R. fusiform gyrus & 36 & + & - & 44 & -62 & -14 \\
R. lingual gyrus & 35 & + & - & 10 & -88 & -10 \\
L. middle frontal gyrus & 35 & + & - & -50 & 4 & 40 \\
R. superior parietal cortex & 35 & + & - & 28 & -58 & 56 \\
R. middle temporal gyrus & 23 & - & + & 50 & -70 & 8 \\
L. caudate & 22 & + & - & -16 & 2 & 20 \\
L. middle frontal gyrus & 19 & + & - & -44 & $5-$ & 16 \\
R. superior parietal cortex & 18 & + & - & 26 & -70 & 46 \\
R. middle frontal gyrus & 17 & + & - & 40 & 52 & 14 \\
L. orbitofrontal gyrus & 15 & - & + & -8 & 32 & -26 \\
L. middle occipital gyrus & 15 & + & - & -22 & -88 & -14 \\
L. insula & 15 & + & - & -38 & 22 & 4 \\
\hline
\end{tabular}

L., left; R., right.

mance (Reward $>$ Neutral; $\left.t_{(293)}=1.9856 ; p=0.05\right)$. Specifically, greater activation of visual association cortex in response to a cue was related to greater benefits of reward motivation on performance. No such relationships were seen with middle temporal gyrus $(p=0.94)$ or OFC $(p=0.85)$.

\section{Discussion}

Previous research has demonstrated enhanced motivation in adolescents (Somerville et al., 2010; Geier, 2013; Padmanabhan and Luna, 2014; Shulman et al., 2016). Rodent research supports that increased reward motivation results from increased eventevoked firing and prolonged baseline engagement of mesolimbic dopamine systems (Bjork et al., 2004; Simon and Moghaddam, 2015). In the current study, we characterized age-related trajectories of state-dependent coupling between the VTA and the NAcc, a proxy of prolonged mesolimbic engagement. In a longitudinal neuroimaging sample, we found context-specific changes in VTA-NAcc circuit development. In motivation contexts (defined as a period where individuals translated incentive cues into goal-oriented behavior), there was increased coupling between the VTA and NAcc that was greatest in preadolescence/early adolescence and decreased into early adulthood. During resting state, there were no age-related differences. These findings extend prior literature on heightened motivation during the adolescent period by adding a novel characterization of state-dependent engagement.

\section{State-dependent changes in adolescent neurodevelopment}

Consistent with prior animal models, we found state-dependent, age-related trajectories of VTA-NAcc functional coupling. Compared with adults, preadolescent and adolescent individuals showed heightened connectivity that was unique to a motivationally relevant context in which they performed an antisaccade task where cues indicated the value of exerting cognitive control. By using a background connectivity analysis technique, we were able to characterize changes in functional coupling that extended over the entire task state, rather than transient engagement in response to individual cues, instantiation of cognitive control, or reward outcomes.
These findings dovetail well with an emerging body of animal literature investigating how the mesolimbic dopamine system contributes to reward sensitivity. Prior animal work has shown that dopamine receptor availability as well as baseline firing rate of dopamine neurons are enhanced in adolescent rodents (Wahlstrom et al., 2010a,b), both of which are putative neurobiological mechanisms of enhanced motivation (Lisman and Grace, 2005; Luo et al., 2011). Our finding of developmental changes in VTANAcc coupling was specific to a motivationally relevant context. When queried during a resting-state context, in which there were no task demands or goals, there were no age-related differences. Our findings support a model of neurodevelopment in which the core structure of mesolimbic circuitry matures relatively early but in the context of motivation connectivity within this circuit is enhanced during the preadolescent/adolescent period. Surprisingly, our modeling indicated that the peak of VTA-NAcc coupling was apparent during the preadolescent period (10-11 year olds), which differs from prior literature demonstrating peaks unique to adolescence (Padmanabhan and Luna, 2014; Silverman et al., 2015). Importantly, our results may be underspecified to accurately detect differences across the preadolescent and early adolescent periods, however, because the current sample undersampled the preadolescent period and did not sample earlier into childhood. Regardless, our findings suggest that although the ability to engage the mesolimbic system is available by adolescence, in the context of reward pursuit or punishment avoidance the adolescent mesolimbic systems may operate at elevated levels.

A limitation of the current study is that comparisons were made across an incentivized task-related context and rest, as opposed to incentivized and non-incentivized task contexts. Given this, our findings currently cannot disambiguate whether contextspecific neurodevelopment of VTA-NAcc coupling is specific to incentivized contexts or rather generalize to any contexts where individuals are viewing task-relevant stimuli and/or executing goaloriented behavior. Given prior research implicating engagement of this circuit during non-incentivized behavior (Kehagia et al., 2010; Ranganath and Jacob, 2016; Boot et al., 2017), we expect that similar developmental trajectories shown in the current study will be present during non-incentivized contexts, albeit to a lesser extent.

\section{Moderators of state-dependent neurodevelopment}

Previous research has demonstrated that the detection of behavioral cues by cortex regulate the functional engagement of the VTA (Murty et al., 2017) and VTA-NAcc coupling (Ballard et al., 2011) and that phasic engagement of the VTA can lead to prolonged activation of mesolimbic systems (Lohani et al., 2018). Given that adolescence may be associated with enhanced sensitivity to goal-relevant information in the environment, we characterized relationships between cue-related activation and our state-dependent increases in VTA-NAcc coupling. We found that cue-evoked activation in OFC, middle temporal gyrus, and visual association cortex were related to age-related decreases in VTA-NAcc coupling. Given its well validated role in value processing (Kringelbach, 2005; de la Vega et al., 2016), we were particularly interested that this whole-brain analysis identified the OFC. Specifically, we found that increased activation in the OFC in response to goal-relevant cues in adolescence partially explained age-related decreases in VTA-NAcc coupling. Prior work in human neuroimaging reliably implicates the OFC in encoding reward value (Levy and Glimcher, 2012). Furthermore, neuroanatomical studies have characterized direct excitatory connections of the medial prefrontal cortex, including the OFC, to the VTA (Cavada et al., 2000; Sesack and Grace, 2010) and have 
A Ventral Visual Stream

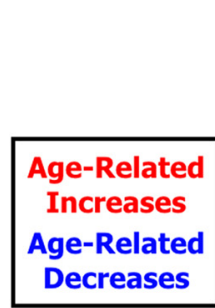

B Orbito-frontal Cortex
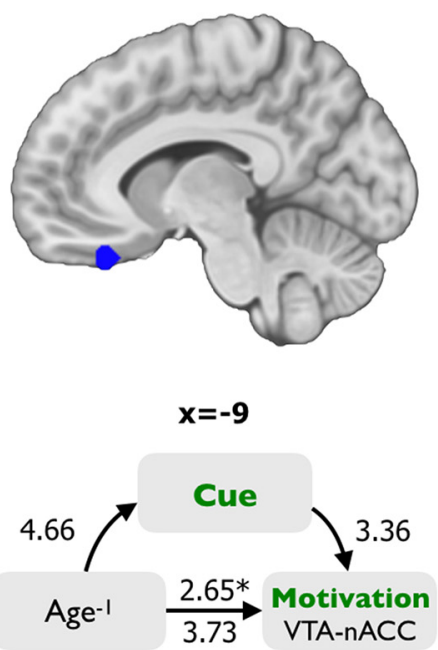

C Middle Temporal Gyrus
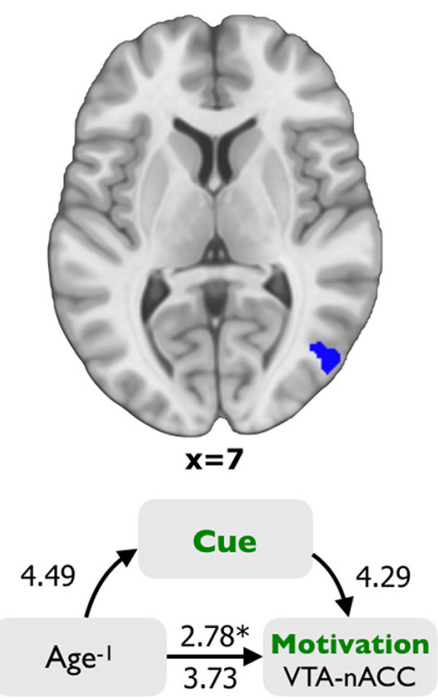

Figure 2. Mediation analysis of age-related decreases in VTA-NAcc coupling. Mediation analysis of cue-related activity identified three regions that partially mediated age-related decreases in VTA-NAcc functional coupling, including age-related increases in the left visual association cortex $(\boldsymbol{A})$ as well as age-related decreases in 0FC (B) and middle temporal gyrus ( $\boldsymbol{C}$.

Table 3. Across-subject correlations among cue-evoked activation in regions identified as partial mediators between age and VTA-NAcc coupling in motivational contexts

\begin{tabular}{lll}
\hline Regions & $R^{2}$ value & $p$ value \\
\hline Visual association cortex-middle temporal gyrus & 0.04 & 0.001 \\
Visual association cortex-orbitofrontal cortex & 0.004 & 0.262 \\
Orbitofrontal cortex-middle temporal gyrus & 0.02 & 0.007 \\
\hline
\end{tabular}

shown that engagement of medial prefrontal cortex can stimulate dopaminergic activation (Murase et al., 1993; Taber and Fibiger, 1993; You et al., 1998). We hypothesize that increased OFC activity in adolescence represents heightened sensitivity to valuerelated cues in the environment, which in turn increases VTANAcc coupling across the entire motivation context, a process that could be associated with greater integration between cortical and subcortical structures (Luna et al., 2015).

Whereas we characterized age-related decreases in OFC engagement to all motivationally relevant cues, prior research has characterized age-related increases in the OFC in response to reward versus neutral events compared with adults (Geier et al., 2010; Van Leijenhorst et al., 2010). Our results may differ because of how we characterized OFC reactivity, because we collapsed cue-related responses across a range of motivation cues that indicated reward, punishment, and neutral incentives. This pattern of findings suggests that the OFC shows less selectivity in adolescence because they have elevated responses to all motivationally relevant cues, whereas in adulthood OFC responses may be specific to reward cues. In fact, when we analyzed our data using comparisons of cue-related response to reward versus neutral/ punishment events, we did not see any significant mediation by the OFC. Thus, we hypothesize that our findings may represent that the engagement of the OFC in adolescents may represent an overgeneralized response to motivation extending to neutral cues, which may be increased because of elevated mesolimbic functioning in the broader context.

Less is known about direct roles of the middle temporal gyrus and visual association cortex in modulating mesolimbic activation, particularly during adolescence. A previous study in healthy adults implicated the middle temporal gyrus is assessing risk during reward-based decision-making (Guo et al., 2013). Furthermore, research has shown protracted maturation of visual association cortex during a spatial working memory task (Simmonds et al., 2017), which in our task may indirectly reflect adolescents' difficulty in reliably executing cognitive control during the antisaccade task (Ordaz et al., 2013). Given these findings, we predict that cue-based responses in these regions may signal a need to further engage mesolimbic systems to successfully earn rewards and avoid punishments. This interpretation would fall in line with prior research, which has shown that engagement of mesolimbic circuitry may underlie adolescents' ability to perform cognitive control tasks at adult levels of performance in the context of reward (Geier et al., 2010). However, future research is needed to directly relate engagement of middle temporal gyrus and visual association cortex in supporting adolescents' ability to successfully implement cognitive control during states of high motivation.

\section{Conclusions}

Together, our findings provide novel evidence that in motivationally relevant contexts there is heightened engagement of mesolimbic circuitry during adolescence that may emerge during the preadolescent period. These findings extend emerging models of adolescent development suggesting that state-dependent increases in dopamine signaling may underlie heightened reward sensitivity. In addition, we provide a network-level model suggesting that elevated mesolimbic processing may be triggered by the processing of motivationally relevant cues in the OFC, middle temporal gyrus, and visual association cortex. Although untested, we predict that engagement of these systems may promote the ability for adolescents to compensate for limitations in the generation of responses that ensure reward receipt. Thus, our findings provide a network-level model of heightened mesolimbic engagement during adolescence, in which adolescents show greater sensitivity to goal-relevant cues in rewarding contexts that in turn increases state-dependent increases in VTA-NAcc coupling. 


\section{References}

Al-Aidroos N, Said CP, Turk-Browne NB (2012) Top-down attention switches coupling between low-level and high-level areas of human visual cortex. Proc Natl Acad Sci U S A 109:14675-14680. CrossRef Medline

Ballard IC, Murty VP, Carter RM, MacInnes JJ, Huettel SA, Adcock RA (2011) Dorsolateral prefrontal cortex drives mesolimbic dopaminergic regions to initiate motivated behavior. J Neurosci 31:10340-10346. CrossRef

Bjork JM, Knutson B, Fong GW, Caggiano DM, Bennett SM, Hommer DW (2004) Incentive-elicited brain activation in adolescents: similarities and differences from young adults. J Neurosci 24:1793-1802. CrossRef

Boot N, Baas M, van Gaal S, Cools R, De Dreu CKW (2017) Creative cognition and dopaminergic modulation of fronto-striatal networks: integrative review and research agenda. Neurosci Biobehav Rev 78:13-23. CrossRef Medline

Cavada C, Compañy T, Tejedor J, Cruz-Rizzolo RJ, Reinoso-Suárez F (2000) The anatomical connections of the macaque monkey orbitofrontal cortex. A review. Cereb Cortex 10:220-242. Medline

Chein J, Albert D, O’Brien L, Uckert K, Steinberg L (2011) Peers increase adolescent risk taking by enhancing activity in the brain's reward circuitry. Dev Sci 14:F1-10. CrossRef Medline

de la Vega A, Chang LJ, Banich MT, Wager TD, Yarkoni T (2016) Largescale meta-analysis of human medial frontal cortex reveals tripartite functional organization. J Neurosci 36:6553-6562. CrossRef

Duncan K, Tompary A, Davachi L (2014) Associative encoding and retrieval are predicted by functional connectivity in distinct hippocampal area CA1 pathways. J Neurosci 34:11188-11198. CrossRef

Frazier JA, Chiu S, Breeze JL, Makris N, Lange N, Kennedy DN, Herbert MR, Bent EK, Koneru VK, Dieterich ME, Hodge SM, Rauch SL, Grant PE, Cohen BM, Seidman LJ, Caviness VS, Biederman J (2005) Structural brain magnetic resonance imaging of limbic and thalamic volumes in pediatric bipolar disorder. Am J Psychiatry 162:1256-1265. CrossRef Medline

Geier CF (2013) Adolescent cognitive control and reward processing: implications for risk taking and substance use. Horm Behav 64:333-342. CrossRef Medline

Geier CF, Terwilliger R, Teslovich T, Velanova K, Luna B (2010) Immaturities in reward processing and its influence on inhibitory control in adolescence. Cereb Cortex 20:1613-1629. CrossRef Medline

Grace AA (2016) Dysregulation of the dopamine system in the pathophysiology of schizophrenia and depression. Nat Rev Neurosci 17:524-532. CrossRef Medline

Grace AA, Floresco SB, Goto Y, Lodge DJ (2007) Regulation of firing of dopaminergic neurons and control of goal-directed behaviors. Trends Neurosci 30:220-227. CrossRef Medline

Guo Z, Chen J, Liu S, Li Y, Sun B, Gao Z (2013) Brain areas activated by uncertain reward-based decision-making in healthy volunteers. Neural Regen Res 8:3344-3352. Medline

Haber SN, Knutson B (2010) The reward circuit: linking primate anatomy and human imaging. Neuropsychopharmacology 35:4-26. CrossRef

Hallquist MN, Hwang K, Luna B (2013) The nuisance of nuisance regression: spectral misspecification in a common approach to resting-state fMRI preprocessing reintroduces noise and obscures functional connectivity. Neuroimage 82:208-225. CrossRef Medline

Hawes SW, Chahal R, Hallquist MN, Paulsen DJ, Geier CF, Luna B (2017) Modulation of reward-related neural activation on sensation seeking across development. Neuroimage 147:763-771. CrossRef Medline

Jalbrzikowski M, Larsen B, Hallquist MN, Foran W, Calabro F, Luna B (2017) Development of white matter microstructure and intrinsic functional connectivity between the amygdala and ventromedial prefrontal cortex: associations with anxiety and depression. Biol Psychiatry 82:511521. CrossRef Medline

Kehagia AA, Murray GK, Robbins TW (2010) Learning and cognitive flexibility: frontostriatal function and monoaminergic modulation. Curr Opin Neurobiol 20:199-204. CrossRef Medline

Kringelbach ML (2005) The human orbitofrontal cortex: linking reward to hedonic experience. Nat Rev Neurosci 6:691-702. CrossRef Medline

Levy DJ, Glimcher PW (2012) The root of all value: a neural common currency for choice. Curr Opin Neurobiol 22:1027-1038. CrossRef Medline
Lisman JE, Grace AA (2005) The hippocampal-VTA loop: controlling the entry of information into long-term memory. Neuron 46:703-713. CrossRef Medline

Lohani S, Martig AK, Underhill SM, DeFrancesco A, Roberts MJ, Rinaman L, Amara S, Moghaddam B (2018) Burst activation of dopamine neurons produces prolonged post-burst availability of actively released dopamine. Neuropsychopharmacology. Advance online publication. Retrieved June 20, 2018. doi:10.1038/s41386-018-0088-7

Luna B, Marek S, Larsen B, Tervo-Clemmens B, Chahal R (2015) An integrative model of the maturation of cognitive control. Annu Rev Neurosci 38:151-170. CrossRef Medline

Luo AH, Tahsili-Fahadan P, Wise RA, Lupica CR, Aston-Jones G (2011) Linking context with reward: a functional circuit from hippocampal CA3 to ventral tegmental area. Science 333:353-357. CrossRef Medline

Marek S, Hwang K, Foran W, Hallquist MN, Luna B (2015) The contribution of network organization and integration to the development of cognitive control. PLoS Biol 13:e1002328. CrossRef Medline

Murase S, Grenhoff J, Chouvet G, Gonon FG, Svensson TH (1993) Prefrontal cortex regulates burst firing and transmitter release in rat mesolimbic dopamine neurons studied in vivo. Neurosci Lett 157:53-56. CrossRef Medline

Murty VP, Shermohammed M, Smith DV, Carter RM, Huettel SA, Adcock RA (2014) Resting state networks distinguish human ventral tegmental area from substantia nigra. Neuroimage 100:580-589. CrossRef Medline

Murty VP, Calabro F, Luna B (2016) The role of experience in adolescent cognitive development: integration of executive, memory, and mesolimbic systems. Neurosci Biobehav Rev 70:46-58. CrossRef Medline

Murty VP, Ballard IC, Adcock RA (2017) Hippocampus and prefrontal cortex predict distinct timescales of activation in the human ventral tegmental area. Cereb Cortex 27:1660-1669. CrossRef Medline

Norbury A, Husain M (2015) Sensation-seeking: dopaminergic modulation and risk for psychopathology. Behav Brain Res 288:79-93. CrossRef Medline

Norman-Haignere SV, McCarthy G, Chun MM, Turk-Browne NB (2012) Category-selective background connectivity in ventral visual cortex. Cereb Cortex 22:391-402. CrossRef Medline

Ordaz SJ, Foran W, Velanova K, Luna B (2013) Longitudinal growth curves of brain function underlying inhibitory control through adolescence. J Neurosci 33:18109-18124. CrossRef

Padmanabhan A, Luna B (2014) Developmental imaging genetics: linking dopamine function to adolescent behavior. Brain Cogn 89:27-38. CrossRef Medline

Patel AX, Kundu P, Rubinov M, Jones PS, Vértes PE, Ersche KD, Suckling J, Bullmore ET (2014) A wavelet method for modeling and despiking motion artifacts from resting-state fMRI time series. Neuroimage 95:287304. CrossRef Medline

Paulsen DJ, Hallquist MN, Geier CF, Luna B (2015) Effects of incentives, age, and behavior on brain activation during inhibitory control: a longitudinal fMRI study. Dev Cogn Neurosci 11:105-115. CrossRef Medline

Ranganath A, Jacob SN (2016) Doping the mind: dopaminergic modulation of prefrontal cortical cognition. Neuroscientist 22:593-603. Medline

Sesack SR, Grace AA (2010) Cortico-basal ganglia reward network: microcircuitry. Neuropsychopharmacology 35:27-47. CrossRef Medline

Shohamy D, Adcock RA (2010) Dopamine and adaptive memory. Trends Cogn Sci 14:464-472. CrossRef Medline

Shulman EP, Smith AR, Silva K, Icenogle G, Duell N, Chein J, Steinberg L (2016) The dual systems model: review, reappraisal, and reaffirmation. Dev Cogn Neurosci 17:103-117. CrossRef Medline

Silverman MH, Jedd K, Luciana M (2015) Neural networks involved in adolescent reward processing: an activation likelihood estimation metaanalysis of functional neuroimaging studies. Neuroimage 122:427-439. CrossRef Medline

Simmonds DJ, Hallquist MN, Luna B (2017) Protracted development of executive and mnemonic brain systems underlying working memory in adolescence: a longitudinal fMRI study. Neuroimage 157:695-704. CrossRef Medline

Simon NW, Moghaddam B (2015) Neural processing of reward in adolescent rodents. Dev Cogn Neurosci 11:145-154. CrossRef Medline 
Somerville LH, Jones RM, Casey BJ (2010) A time of change: behavioral and neural correlates of adolescent sensitivity to appetitive and aversive environmental cues. Brain Cogn 72:124-133. CrossRef Medline

Taber MT, Fibiger HC (1993) Electrical stimulation of the medial prefrontal cortex increases dopamine release in the striatum. Neuropsychopharmacology 9:271-275. CrossRef

Telzer EH (2016) Dopaminergic reward sensitivity can promote adolescent health: a new perspective on the mechanism of ventral striatum activation. Dev Cogn Neurosci 17:57-67. CrossRef Medline

van Duijvenvoorde AC, Op de Macks ZA, Overgaauw S, Gunther Moor B, Dahl RE, Crone EA (2014) A cross-sectional and longitudinal analysis of reward-related brain activation: effects of age, pubertal stage, and reward sensitivity. Brain Cogn 89:3-14. CrossRef Medline

Van Leijenhorst L, Zanolie K, Van Meel CS, Westenberg PM, Rombouts
SARB, Crone EA (2010) What motivates the adolescent? Brain regions mediating reward sensitivity across adolescence. Cereb Cortex 20:61-69. CrossRef Medline

Wahlstrom D, Collins P, White T, Luciana M (2010a) Developmental changes in dopamine neurotransmission in adolescence: behavioral implications and issues in assessment. Brain Cogn 72:146-159. CrossRef Medline

Wahlstrom D, White T, Luciana M (2010b) Neurobehavioral evidence for changes in dopamine system activity during adolescence. Neurosci Biobehav Rev 34:631-648. CrossRef Medline

You ZB, Tzschentke TM, Brodin E, Wise RA (1998) Electrical stimulation of the prefrontal cortex increases cholecystokinin, glutamate, and dopamine release in the nucleus accumbens: an in vivo microdialysis study in freely moving rats. J Neurosci 18:6492-6500. CrossRef 